\title{
Vector Boson Scattering at High Mass
}

\section{Peter Sherwood*}

University College London

on behalf of the ATLAS Collaboration

E-mail: peter.sherwoodecern.ch

Physics at LHC 2008

29 September - October 4, 2008

Split, Croatia

\footnotetext{
${ }^{*}$ Speaker.
} 


\section{Introduction}

In the Standard Model, vector boson scattering (VBS) amplitudes violate unitarity for $\mathrm{m}_{H}>$ $870 \mathrm{GeV}$ [1]. If a light Higgs Boson does not exist, some new physics must be at work. Vector boson scattering resonances (see fig 1) could be a possible manifestation of this new physics. Such resonances are predicted by a number of theories $[2,3,4,5,6,7,8,9]$.

All of these theories are described by the Electro-weak Chiral Langrangian (EWCL) [10, 11], which is an effective theory valid for energies up to $\approx 3 \mathrm{TeV}$. At LHC energies it is necessary to unitarise the scattering amplitudes. The calculated resonance structure is affected by the unitarisation scheme used. Here ${ }^{1}$, we show results found using the Inverse Amplitude (Padé) unitarisation scheme [13].

The EWCL has a small number of parameters, two of which ( $a_{4}$ and $a_{5}$, see fig 2 ) describe VBS. These two parameters are limited to be $<10^{-2}$ by low energy data. Poles can occur in the physical part of the plane, which correspond to resonances. These can be either scalar Higgs Boson like resonances, or vector technicolor-like resonances [14].

\section{Signals and Backgrounds}

Signal events were generated with $\mathrm{a}_{4}$ and $\mathrm{a}_{5}$ set to values which give rise to resonances at or around 500, 800 and $1100 \mathrm{GeV}$. WW, WZ and ZZ interactions were investigated.

These events are characterised by:

- Tag jets at high $|\eta|$ (see fig 3)

- Absence of hadronic activity between tag jets due to the color-free VBS interaction.

- The presence of two vector bosons. In this study, one boson was required to decay leptonically, and the other to decay hadronically. For highly boosted hadronically decaying bosons, the two jets often overlap, and are not resolved by the jet finders.

\footnotetext{
${ }^{1}$ For a fuller discussion, see [12]
}

Figure 1: VBS scattering: the scattered quarks give rise to the forward tag jets. There is no color connection between the outgoing forward quarks, and the central region has little hadronic activity.

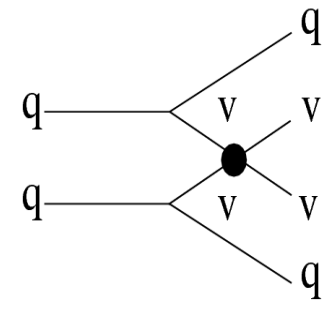


The signal event samples were generated using Pythia 6.403 [15] modified to use the amplitudes of Dobado et al [16] which uses the EWCL and the Inverse Amplitude unitarisation scheme. CTEQ6L parton density functions where used with renormalisation scale $\mathrm{Q}^{2}=M_{W}^{2}$.

Backgrounds to the signal include:

- $\mathrm{Z}$ or $\mathrm{W}+3$ jets (background to events where the two jets are not resolved).

- $\mathrm{Z}$ or $\mathrm{W}+4$ jets (background to events where the two jets are resolved).

- $\mathrm{t} \overline{\mathrm{t}}$

The vector boson + jets events were generated using MADGRAPH [17]. The parton showering was provided by Pythia 6.403. The renormalisation and refactorisation scale were given by $\mathrm{Q}^{2}=$ $M_{W}^{2}$. The $\bar{t}$ events were generated using MC@NLO [18, 19] with Herwig [20, 21] used for the parton shower hadronisation, and JIMMY $[22,23]$ used for the underlying event.

The generated events were passed through the full ATLAS detector simulation and event reconstruction chain.

\section{Event Selection}

\subsection{Leptonic Vector Boson Reconstruction}

- The decay $W^{ \pm} \rightarrow \ell^{ \pm} v$ was reconstructed using the nominal value for $M_{W}$, the charged lepton momentum $\mathbf{p}_{\ell}$, and by taking the $\mathbf{p}_{\mathbf{t}}$ of the missing momentum of the event as $\mathbf{p}_{\mathbf{t}}^{v}$. If the resulting quadratic expression in $p_{z}^{v}$ had a real solution, the event was said to contain a $\mathrm{W}$.

- $Z \rightarrow \ell^{+} \ell^{-}$decays were reconstructed from same flavor, oppositely charged leptons. Momentum and $|\eta|$ cuts were applied to the leptons, and a mass cut was applied to the lepton pair.

\subsection{Hadronic Vector Boson Reconstruction}

Jets were reconstructed using both cone and $k_{\perp}[24,25,26]$ jet finders. Hadronically decaying vector bosons which are decay products of light $(\approx 500 \mathrm{GeV}$ and below) resonances usually decay to two distinct jets. As the resonance mass increases, the jets overlap, and cannot be resolved directly by the jet finders. However, it was possible to exploit the sub-jet structure of the resulting mono-jet to retrieve the two overlapping jets. Using the results of the $k_{\perp}$ algorithm, it is expected that, for the final merge, $Y \equiv E_{T} \times \sqrt{y} \approx m_{V} / 2$ where $y$ is the recombination parameter. For light

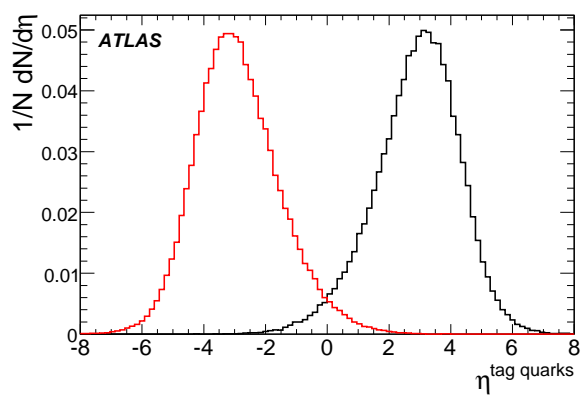

Figure 3: $\eta$ distribution for quarks giving rise to the tag jets. jets, $\mathrm{Y}$ is expected to be much lower [27]. Figure 4 gives the fraction of single jet $\mathrm{W}$ candidates as a function of $p_{t}$ for a high mass resonance. 


\begin{tabular}{|c|c|c|c|c|}
\hline Process & resonance & \multicolumn{2}{|c|}{ cross section $(\mathrm{fb})$} & Luminosity \\
\cline { 3 - 4 } & mass $(\mathrm{GeV})$ & signal & background & ${\text { for } 5 \sigma\left(\mathrm{fb}^{-1}\right)}$ \\
\hline$W W / W Z \rightarrow \ell v j j$ & 500 & $0.31 \pm 0.05$ & $0.79 \pm 0.26$ & 235 \\
\hline$W W / W Z \rightarrow \ell v j j$ & 800 & $0.65 \pm 0.04$ & $0.87 \pm 0.28$ & 60 \\
\hline$W W / W Z \rightarrow \ell v j j$ & 1100 & $0.24 \pm 0.03$ & $0.46 \pm 0.25$ & 230 \\
\hline$W_{j j} Z_{\ell \ell}$ & 500 & $0.28 \pm 0.04$ & $0.20 \pm 0.18$ & 90 \\
\hline$W_{\ell v} Z_{\ell \ell}$ & 500 & $0.40 \pm 0.03$ & $0.25 \pm 0.03$ & 55 \\
\hline$W_{j j} Z_{\ell \ell}$ & 800 & $0.24 \pm 0.02$ & $0.30 \pm 0.22$ & 160 \\
\hline$W_{j} Z_{\ell \ell}$ & 800 & $0.20 \pm 0.02$ & $0.09 \pm 0.06$ & 90 \\
\hline$W_{j} Z_{\ell \ell}$ & 1100 & $0.11 \pm 0.01$ & $0.10 \pm 0.06$ & 250 \\
\hline$W_{\ell v} Z_{\ell \ell}$ & 1100 & $0.070 \pm 0.004$ & $0.020 \pm 0.009$ & 200 \\
\hline$Z_{v v} Z_{\ell \ell}$ & 500 & $0.32 \pm 0.02$ & $0.15 \pm 0.03$ & 60 \\
\hline
\end{tabular}

Table 1: Approximate signal and background cross sections expected after the analysis event selections, and the approximate value of the integrated luminosity required for a $5 \sigma$ significance. The uncertainties are due to Monte Carlo statistics only.

\subsection{VB Kinematic Cuts}

The vector bosons were required to have $p_{t}>200 \mathrm{GeV}$, and $|\eta|<2$

\subsection{Tag Jets, top veto and Central Region Requirements}

The presence of two tag jets with $\mathrm{E}>300 \mathrm{GeV}$ with a large rapidity gap $(|\Delta \eta|>5)$ was required. No jet (other than tag jets and hadronic VB decay jets) with $p_{t}>30 \mathrm{GeV}$ between the tag jets was allowed. A top veto was applied to the channels with a large $t \bar{t}$ background. This veto rejects events in which a $\mathrm{W}$ candidate combines with another jet to give a mass within a window of 130$240 \mathrm{GeV}$.

\section{Results}

Table 1 gives the signal and background

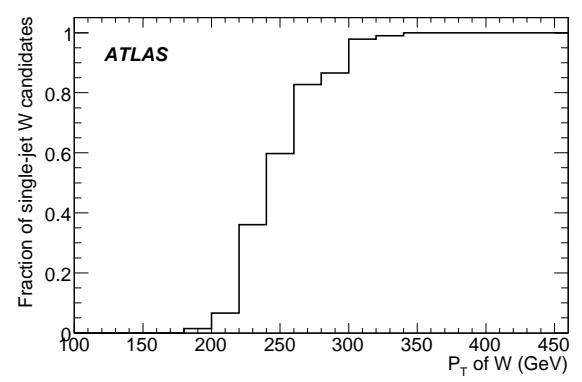

Figure 4: Fraction of single jet hadronic $\mathrm{W}$ candidates originating from the decay of a high mass resonance. cross sections for the three masses investigated, together with the integrated luminosity required to observe a significance of $5 \sigma$.

\section{Summary}

We have performed a study of high mass vector boson scattering events using simulated events. The ATLAS detector response was simulated and the full reconstruction software was used. A subjet analysis was used to disentangle overlapping jets arising from the decays of highly boosted 
vector bosons. The analysis is necessarily a sketch as the backgrounds will need to be evaluated from the data. Nonetheless, we estimate it will take an integrated luminosity in the range 50-250 $\mathrm{fb}^{-1}$ to observe possible high mass resonances arising from vector boson scattering.

\section{References}

[1] S. Dawson, hep-ph/9901280.

[2] K. Lane and S. Mrenna, Phys. Rev. D67, 115011 (2003), [hep-ph/0210299].

[3] C. Csaki, C. Grojean, H. Murayama, L. Pilo and J. Terning, Phys. Rev. D69, 055006 (2004), [hep-ph/0305237].

[4] C. Csaki, C. Grojean, L. Pilo and J. Terning, Phys. Rev. Lett. 92, 101802 (2004), [hep-ph/0308038].

[5] G. Cacciapaglia, C. Csaki, C. Grojean and J. Terning, Phys. Rev. D71, 035015 (2005), [hep-ph/0409126].

[6] C. Csaki, hep-ph/0412339, Talk presented at SUSY 2004, Tsukuba, Japan, June 17-23 2004, arXiv:hep-ph/0412339.

[7] A. Birkedal, K. T. Matchev and M. Perelstein, hep-ph/0508185.

[8] R. Sekhar Chivukula et al., Phys. Rev. D74, 075011 (2006), [hep-ph/0607124].

[9] R. Casalbuoni, S. De Curtis and M. Redi, Eur. Phys. J. C18, 65 (2000), [hep-ph/0007097].

[10] W. Kilian, Springer Tracts Mod. Phys. 198, 1 (2003).

[11] W. Kilian, hep-ph/0303015.

[12] G. Aad et al., CERN-OPEN-2008-020.

[13] A. Dobado and J. R. Pelaez, Phys. Rev. D56, 3057 (1997), [hep-ph/9604416].

[14] A. Dobado, M. J. Herrero, J. R. Pelaez and E. Ruiz Morales, Phys. Rev. D62, 055011 (2000), [hep-ph/9912224].

[15] T. Sjostrand, S. Mrenna and P. Skands, JHEP 05, 026 (2006), [hep-ph/0603175].

[16] A. Dobado, M. J. Herrero and J. Terron, Z. Phys. C50, 465 (1991).

[17] F. Maltoni and T. Stelzer, JHEP 02, 027 (2003), [hep-ph/0208156].

[18] S. Frixione and B. R. Webber, JHEP 06, 029 (2002), [hep-ph/0204244].

[19] S. Frixione, P. Nason and B. R. Webber, JHEP 08, 007 (2003), [hep-ph/0305252].

[20] G. Corcella et al., hep-ph/0210213.

[21] G. Corcella et al., JHEP 01, 010 (2001), [hep-ph/0011363].

[22] J. M. Butterworth, J. R. Forshaw and M. H. Seymour, Z. Phys. C72, 637 (1996), [hep-ph/9601371].

[23] S. Alekhin et al., hep-ph/0601012.

[24] S. Catani, Y. L. Dokshitzer, M. H. Seymour and B. R. Webber, Nucl. Phys. B406, 187 (1993).

[25] J. M. Butterworth, J. P. Couchman, B. E. Cox and B. M. Waugh, Comput. Phys. Commun. 153, 85 (2003), [hep-ph/0210022].

[26] M. Cacciari and G. P. Salam, Phys. Lett. B641, 57 (2006), [hep-ph/0512210].

[27] J. M. Butterworth, B. E. Cox and J. R. Forshaw, Phys. Rev. D 65 (2002), [hep-ph/0201098]. 